\title{
ENUMERATING SUBGROUPS
}

\section{R. J. COOK, JAMES WIEGOLD and A. G. WILLIAMSON}

(Received 6 November 1985)

Communicated by $\mathrm{H}$. Lausch

\begin{abstract}
It is proved that a finite soluble group of order $n$ has at most $(n-1) /(q-1)$ maximal subgroups, where $q$ is the smallest prime divisor of $n$.
\end{abstract}

1980 Mathematics subject classification (Amer. Math. Soc.): primary 20 D 10; secondary 20 D 99.

\section{Introduction}

Much work has been done on estimating the number of groups of given order $n$ (see P. M. Neumann [2] and the articles cited there), but as far as we know the only appearance in the literature of estimates for the number of subgroups of a group of given order $n$ are those of P. Hall [1] relating to $p$-groups. In this note we shall derive estimates for the number of different maximal subgroups of a finite soluble group of order $n$. In particular, there are at most $(n-1) /(q-1)$ different maximal subgroups, where $q$ is the smallest prime divisor of $n$, and this bound is attained only for elementary abelian $q$-groups. These results may be applied in an obvious manner to give upper bounds for the total number of subgroups of a soluble group of order $n$. We believe that our results hold for insoluble groups as well, but there appears to be no easy way of verifying this.

As to lower bounds, it is elementary to show that every group of order $n$ has at least $1+\lambda(n)$ subgroups, where $\lambda(n)$ is the number of prime factors of $n$,

(C) 1987 Australian Mathematical Society $0263-6115 / 87 \$ A 2.00+0.00$ 
counted with multiplicity. The only groups with so few subgroups are the cyclic p-groups.

\section{Maximal subgroups of soluble groups}

Every finite soluble group $G$ has a minimal normal subgroup of prime-power order $p^{r}$. By a result of Remak [3], the product $L$ of all such subgroups of order $p^{r}$ is a direct product of some of them:

$$
L=N_{1} \times N_{2} \times \cdots \times N_{k} .
$$

Proposition 2.1. The number of maximal subgroups of $G$ not containing $N_{1}$ is at most $\left|O_{p}(G)\right|$.

Proof. Here, as usual, $O_{p}(G)$ denotes the maximal normal $p$-subgroup of $G$. We proceed by induction on $s$, where $\left|O_{p}(G)\right|=p^{s}$. The proof divides naturally into the two cases $k=1, k>1$.

When $k=1$, we use the well-known result that all maximal subgroups $T$ not containing $N_{1}$ are conjugate. There are $\left|G: N_{G}(T)\right| \leqslant|G: T|=p^{r}$ of them, and this establishes the result in this case. In particular, it is true if $s=1$. Notice that equality is attained only if $T$ is self-normalizing, so that the inequality is strict when $G$ is nilpotent.

Suppose now that $s>1$, that $k>1$, and that the proposition holds for smaller values of $s$. We shall show that every maximal subgroup $T$ not containing $N_{1}$ contains a normal elementary abelian subgroup of order $p^{r}$, and this will enable us to set up an inductive procedure establishing the result.

Write $H=\left(N_{1} \times N_{2}\right) \cap T$. Then $H$ is normal in $G$ since $N_{1} \times N_{2}$ is abelian, and since $G=N_{1} T=\left(N_{1} \times N_{2}\right) T$ and $N_{1} \cap T=1$, we have

$$
\left|N_{1} T\right|=\left|N_{1} \times N_{2}\right||T| /|H|,
$$

so that $|H|=p^{r}$. Thus $T$ contains a normal elementary abelian subgroup of order $p^{r}$, as claimed. Let $\left\{M_{i}: 1 \leqslant i \leqslant b\right\}$ be the set of normal subgroups of order $p^{r}$ contained in $N_{1} \times N_{2}$. To see how large $b$ can be, we argue as follows. We have that $M_{i} \cap M_{j}=1$ if $i \neq j$, since a normal subgroup of $N_{1} \times N_{2}$ of order greater than $p^{r}$ must be the whole of $N_{1} \times N_{2}$. Then $\left|\cup M_{i}\right|=b\left(p^{r}-1\right)+1$, while $\cup M_{i} \subseteq N_{1} \times N_{2}$, and so $b \leqslant p^{r}+1$.

To complete the proof of the proposition, we need some more terminology. We may take $M_{1}=N_{1}$. For $i \neq 1$, let $m_{i}$ be the number of maximal subgroups of $G$ which contain $M_{i}$ but not $N_{1}$. Note that $s>r$ since $k>1$. The arguments above 
show that the number of maximal subgroups of $G$ not containing $N_{1}$ is at most

$$
\sum_{i \neq 1} m_{i} \leqslant(b-1) \max _{i \neq 1} m_{i} \leqslant p^{r} \max _{i \neq 1} m_{i} .
$$

However, $m_{i}$ is the number of maximal subgroups in the factor-group $G / M_{i}$ which do not contain $\left(N_{1} \times M_{i}\right) / M_{i}=\left(N_{1} \times N_{2}\right) / M_{i}$. Since $\left(N_{1} \times M_{i}\right) / M_{i}$ is a minimal normal subgroup of order $p^{r}$ in $G / M_{i}$, and since $O_{p}\left(G / M_{i}\right)$ has order $p^{s-r}$, the induction hypothesis implies that $m_{i} \leqslant p^{s-r}$. Hence we may conclude that the number of maximal subgroups of $G$ not containing $N_{1}$ is at most $p^{r} p^{s-r}=p^{s}$, as required.

It is well-known that the number of maximal subgroups of a $d$-generator $p$-group is exactly $\left(p^{d}-1\right) /(p-1)$, and that this bound is achieved only for elementary abelian groups (for which $d=n$ ). Thus the bound given in the next result is sharp.

THEOREM 2.2. Let $G$ be a finite soluble group of order $n$ and $q$ the smallest prime factor of $n$. Then $G$ has not more than $(n-1) /(q-1)$ different maximal subgroups. This bound is achieved if and only if $G$ is elementary abelian.

Proof. We proceed by induction on $n$. The result is true for groups of small order, and furthermore we may assume that $G$ is not of prime-power order. Let $N$ be a minimal normal subgroup, of order $p^{r}$ say, and write $n=p^{s} t$, where $p^{s}=\left|O_{p}(G)\right|$.

First suppose that $q \neq p$, so that $p>q$. By Proposition 2.1, the number of maximal subgroups not containing $N$ is at most $p^{s}$. By induction, the number that do contain $N$ is at most $\left(n / p^{r}-1\right) /(q-1)$. The (strict) bound $(n-1) /(q-1)$ follows unless $q p^{s}-p^{s}+n / p^{r} \geqslant n$. In fact this inequality cannot happen. If it did, then, since $q p^{s}$ divides $n$, it would be the case that $q p^{s}=n$. But then

$$
q p^{s}-p^{s}+n / p^{r}=n-p^{s}+q p^{s-r}=n-p^{s-r}\left(p^{r}-q\right)<n-1,
$$

which is a contradiction.

Finally, suppose that $p=q$. We establish a chain of inequalities. Since $G$ is not a $p$-group, we have $p^{s+1}<n$, so that $p^{s}<n / p$. Thus $p^{s}(p-1)<n(p-1) / p$ and $p^{s}(p-1)+n / p<n$. A fortiori, $p^{s}(p-1)+n / p^{r}-1<n-1$, so that $p^{s}+\left(n / p^{r}-1\right) /(p-1)<(n-1) /(p-1)$.

The argument that completes the proof runs just as before.

The expression $(n-1) /(q-1)$ is a nice general expression, but it is not a particularly good bound when $|G|$ is divisible by several primes. In this situation, the following alternative provides a better estimate. It is achieved by metacyclic groups of order $2 p$. 
THEOREM 2.3. Let $G$ be a finite soluble group of order $n=\prod_{i=1}^{k} p_{i}^{\alpha_{i}}$, where the $p_{i}$ are different primes. Then the number of different maximal subgroups of $G$ is at most

$$
\sum_{i=1}^{k}\left(\frac{p_{i}^{\alpha_{i}+1}-p_{i}}{p_{i}-1}\right)-1=\sum_{i=1}^{k} \sum_{j=1}^{\alpha_{i}} p_{i}^{j}-1 .
$$

The proof proceeds by induction on $n$ and is so similar to our preceding arguments that we feel justified in omitting it.

\section{References}

[1] P. Hall, 'A contribution to the theory of groups of prime-power order', Proc. London Math. Soc. 36 (1933), 29-95.

[2] P. M. Neumann, 'An enumeration theorem for finite groups', Quart. J. Math. Oxford Ser. (2) 20 (1969), 395-401.

[3] R. Remak, 'Über minimale invariante Untergruppen in der Theorie der endlichen Gruppen', $J$. Reine Angew. Math. 162 (1930), 1-16.

Department of Pure Mathematics

The University

Sheffield

England
Department of Pure Mathematics University College

Cardiff

Wales

Haywards Heath College

Haywards Heath

West Sussex

England 